\title{
Desempeño laboral de los trabajadores del área de operaciones de la Clínica Good Hope, Miraflores, 2015: una evaluación de 360 grados Labor performance of the workers of the area of operations of the Clinical Good Hope, Miraflores, 2015: An evaluation of 360 degrees
}

\author{
Lucero Wendy, Baquerizo Crisóstomo*1 \\ ${ }^{1}$ Escuela Profesional de Administración, Facultad de Ciencias Empresariales, Universidad Peruana Unión
}

\section{INFORMACIÓN DEL ARTÍCULO}

Historia del artículo

Recibido: 23 de junio 2016

Aceptado: 12 de octubre del 2016

Palabras clave:

Evaluación de 360 grados, desempeño laboral, conocimiento del trabajo, calidad de trabajo, relaciones con las personas, estabilidad emotiva, capacidad de síntesis analítica.

\section{Keywords:}

Evaluation of 360 degrees, labor performance, knowledge of the work, quality of work, relations with the persons, emotive stability, capacity of analytical synthesis.

\begin{abstract}
Resumen
El objetivo de la investigación fue determinar la percepción del desempeño laboral mediante la evaluación de 360 grados en los trabajadores del área de operaciones de la Clínica Good Hope Miraflores, 2015. La evaluación de 360 grados es una herramienta básica para contar con diferentes perspectivas, tomar buenas decisiones y mejorar la gestión administrativa de una empresa. El tipo de investigación fue cuantitativo con alcance descriptivo. La muestra estuvo conformada por 244 personas compuesta por jefes, ejecutivos, colaboradores, y clientes. Se utilizó el muestreo probabilístico. Los resultados de la evaluación de 360 grados del desempeño laboral, muestra que es aplicable este modelo a la Clínica Good Hope, toda vez que el $71 \%$ de jefes perciben que la evaluación de 360 grados del desempeño laboral es alto, para el $36 \%$ de ejecutivos el desempeño laboral es alto, y para el $54 \%$ de los colaboradores el desempeño laboral es medio.
\end{abstract}

* Autor de correspondencia: Lucero Wendy Baquerizo Crisóstomo Correo elctrónico: bel_a1991@hotmail.com 


\section{Introducción}

Bernárdez (2006) consideró que la labor de un jefe es aprovechar al máximo las habilidades de los empleados que tienen a su cargo en favor de lograr los objetivos de la empresa; por ello, se necesita que estos empleados cumplan con el perfil que requiere cada puesto, que no siempre se garantiza con el currículum vitae, ni por un reporte de objetivos cumplidos; si el empleado no tiene el perfil para un determinado puesto $y$, además, no existe un plan de capacitaciones que permita acortar esta brecha aunque sea muy bueno, no trabajará al $100 \%$ y su desarrollo profesional tomará más tiempo, surgiendo probablemente, a corto plazo, el descontento $y$, por ende, su renuncia o un trabajo rutinario en degrado de los objetivos de la organización.

Lara, Moras, Morales y Galán (2010) realizaron una investigación en una empresa metal-mecánica para conocer el desempeño de los supervisores, utilizando la evaluación de $360^{\circ}$. La metodología propuso aplicar las siguientes etapas: (a) identificación del personal, (b) realización de entrevistas al personal, (c) definición de las competencias cardinales y específicas, (d) operacionalización de las variables, (e) elaboración de un cuestionario con las competencias identificadas, ( $f$ ) aplicación de una prueba piloto y de la evaluación definitiva y (g) análisis e interpretación de los informes de evaluación para su retroalimentación. Bajo esta metodología se evaluaron a ocho trabajadores y los evaluadores fueron; el mismo supervisor, el jefe inmediato, la pareja de trabajo, el compañero, personal externo y subordinado. Una vez identificadas las competencias generales y las competencias específicas, se elaboró el cuestionario para que los evaluadores pudieran contestarlo. Se logró que los evaluados identifiquen y tomen conciencia de sus fortalezas y debilidades en cuanto a las competencias requeridas en su puesto de trabajo, lo que ayudó a aumentar su nivel de desempeño y rendimiento. La empresa notó que había más productividad y menos desperdicio de materia prima.

La evaluación de 360 grados del desempeño laboral es un medio para evaluar el recurso humano y permite tomar buenas decisiones para alcanzar el éxito de una empresa.

Robbins y Decenzo (2002) mencionaron que este método de evaluación de 360 grados busca la retroalimentación de una serie de fuentes con respecto a la persona que está siendo calificada mediante un instrumento de medición; además, es un sistema de apreciación del desempeño del individuo en el cargo y de su potencial desarrollo y competencia.

Considerando la argumentación y antecedentes similares al estudio, a continuación se identifican, en la institución de estudio, las principales situaciones problemáticas, objeto de investigación: 1) no están identificados los factores que comprenden la evaluación del desempeño laboral, 2) no se realizan evaluaciones del desempeño de personal, 3) no se alcanzan los resultados que se esperan, 4) no se han identificado las habilidades y la capacidad de cada individuo que labora en la empresa.

\section{Revisión de la literatura}

\section{Desempeño laboral}

Cuando se habla de desempeño laboral el individuo manifiesta las competencias laborales alcanzadas en las que se integran, como un sistema, conocimientos, habilidades, experiencias, sentimientos, actitudes, motivaciones, características personales y valores que contribuyen a alcanzar los resul- 
tados que se esperan, en correspondencia con las exigencias técnicas productivas y de servicios de la empresa.

Según Chiavenato (2000), el desempeño se define como las acciones o comportamientos observados en los empleados, relevantes para el logro de los objetivos de la organización. Afirmó que un buen desempeño laboral es la fortaleza más relevante con la que cuenta una organización, tal desempeño varía de una persona a otra y dependerá de factores condicionantes que influyen (recompensas, habilidades y esfuerzo); es decir, cada individuo es capaz de determinar su costo-beneficio para saber cuánto vale la pena desarrollar determinada actividad, dependiendo de las habilidades y la capacidad de cada individuo y cómo percibe este, el papel que desempeña. Werther y Davis (1995) definieron las mediciones del desempeño como los sistemas de calificación de cada labor. Estos mismos autores comentan que las mediciones deben ser de uso fácil, confiables y deben calificar los elementos esenciales que determinan el desempeño. Arrigada (2002) mencionó que la medición del desempeño no es un sistema policial de control, más bien es un sistema que permite saber con mayor precisión cuál es la capacidad real de la organización para cumplir con sus metas, con ayuda del conocimiento, experiencia y esfuerzo.

Por su parte Faria (1995), consideró el desempeño laboral como el resultado del comportamiento de los trabajadores frente al contenido de su cargo, sus atribuciones, tareas y actividades, depende de un proceso de mediación o regulación entre él y la empresa. En tal sentido, la estabilidad laboral está asociado a un mejor desempeño, el cual ha sido considerado como elemento fundamental para medir la efectividad y éxito de una organización. La estabilidad laboral genera a la persona tranquilidad, salud, motivación y buen estado emocional. En estas condiciones, el individuo está dispuesto a dar más y enfocarse hacia otros horizontes que lo lleven a mejorar su posición, tanto en la organización como en la sociedad. Por último, Robbins y Coulter (2005), vincula el desempeño con la capacidad de coordinar y organizar las actividades que al integrarse modelan el comportamiento de las personas involucradas en el proceso productivo.

\section{Evaluación del desempeño}

Según Crespo, Mortis y Tapia (2011), establecer una definición de evaluación del desempeño no es sencillo porque cada autor establece sus propios criterios a partir de las concepciones que han existido a lo largo de la historia. Este concepto, según García, (2010) ha estado influenciado por distintas corrientes como a) la administración científica iniciada por Frederick Winslow Taylor (1956-1015), b) la administración industrial y general sostenida por Henry Fayol (1841-1925), c) la Escuela de Relaciones Humanas que tuvo como precursor a George Elton Mayo (1880-1949) y c) la administración por objetivos de Peter Drucker en 1909.

Según Encina (2003), la evaluación del desempeño es útil para: (a) mejorar las actividades de la empresa en razón de selección y capacitación y (b) brindar información a los empleados para que mejoren su rendimiento. Nieto (2003) mencionó que evaluar el desempeño es verificar si el evaluado está cumpliendo con sus funciones y responsabilidades en un tiempo determinado, ver el cumplimiento mediante los resultados propuestos por la organización, para mejorar el desempeño.

González y Olivares (2005) comentaron que en 1989 la Asociación Americana de Psicología encontró 20 posibles aplicacio- 
nes de la evaluación del desempeño, de los cuales a continuación se mencionan las 13 más representativas: 1) administración de salarios, 2) retroalimentación del desempeño, 3) identificación de las fortalezas y debilidades del evaluado, 4) documentación de las decisiones del personal, 5) reconocimiento del desempeño de la persona, (6) determinación de la promoción, (7) identificación del desempeño deficiente, 8) apoyo para la identificación de metas, 9) decisión de retener o despedir, 10) evaluación del alcance de metas, 11) cumplir los requerimientos legales, 12) decisiones sobre despidos y 13) determinar transferencias o asignaciones.

Chiavenato (2001) mencionó que cuando un programa de evaluación del desempeño está bien planeado, coordinado y desarrollado, normalmente trae beneficios a corto, mediano y largo plazo. Los principales beneficiarios son, generalmente, el evaluado, el jefe, la empresa y la comunidad.

De acuerdo a Mondy y Noe (2005), las organizaciones no deberían eliminar las evaluaciones del desempeño porque ofrece importantes beneficios entre ellas la retroalimentación para motivar al empleado a mejorar, tomar las mejores decisiones, justificar las renuncias, conocer las necesidades de capacitación, desarrollo y poder defender las necesidades del recurso humano, más aún en un mercado globalizado en que la empresa procura los mejores resultados para lograr la eficiencia.

Por su parte, Lau (2010) comentó que evaluar puede convertirse en una herramienta de desarrollo para una empresa, esto es debido a que dispone de elementos importantes para la toma de decisiones, y esto permite gestionar lo necesario para ubicar a los evaluados en otros espacios en donde se puedan desarrollar.
Para García (2001), la evaluación del desempeño ayuda a verificar si el personal de una empresa está avanzando o si requiere de una retroalimentación para mejorar su desempeño; también puntualiza que los empleados entrarán a un clima de realización, haciendo su trabajo de la mejor manera.

Hellriegel y Slocum (2005) puntualizaron que durante 50 años se ha hecho diferentes estudios para mejorar la precisión de las evaluaciones del desempeño. Concluyeron que es muy difícil evaluar con precisión el desempeño, sin embargo, hay herramientas 0 estrategias que pueden ayudar a precisar tales evaluaciones, como las siguientes: 1) formato de escala de estimación: donde se registra los juicios del evaluador hacia el evaluado, 2) auxiliares de memoria: que registran de manera regular los comportamientos o resultados de la evaluación, 3) capacitación del estimador: que es la capacitación al evaluador para mejorar las habilidades de observación, 4) recompensas: consiste en recompensar a los evaluadores que hacen una valoración oportuna y justa del evaluado y 5) múltiples estimadores: donde se recomienda una evaluación de 360 grados.

\section{Materiales y métodos}

El enfoque de esta investigación es cuantitativo porque mide las variables de estudio y aplica el análisis estadístico con un alcance descriptivo. Es descriptivo, porque evalúa y estima los niveles de desempeño laboral en la medida que se aplica los factores de evaluación de 360 grados. El enfoque pretende ampliar el conocimiento ya existente, en función de las veces que se mide la variable y, por el momento en que se recoge la información, es trasversal.

La presente es una investigación no experimental de diseño trasversal, puesto 
que este estudio se realiza sin la manipulación deliberada de la variable y en los que solo se observan los fenómenos en su ambiente natural para después analizarlos en un momento único (Kerlinger y Lee, 2002).

\section{Participantes}

La cantidad de los participantes estuvo conformada por 312 entre personal de la clínica y clientes: 200 clientes recurrentes, 66 ejecutivos y/o equipos de trabajo, 7 jefes y 39 colaboradores.

\section{Tabla 1}

Clientes reiterativos por mes

\begin{tabular}{cc}
\hline Meses & $\begin{array}{c}\text { Cantidad de clientes } \\
\text { reiterativos }\end{array}$ \\
\hline Enero & 198 \\
Febrero & 200 \\
Marzo & 205 \\
Abril & 202 \\
Mayo & 194 \\
Junio & 198 \\
Julio & 202 \\
Agosto & 205 \\
Setiembre & 200 \\
\hline Total & 200 \\
\hline
\end{tabular}

Elaboración propia

Nota: Los clientes reiterativos son los pacientes afiliados a la clínica y hacen uso constante de los servicios de salud.

Tabla 2

Segmentación poblacional de los trabajadores

\begin{tabular}{cc}
\hline $\begin{array}{c}\text { Segmentación poblacional de la Clínica Good } \\
\text { Hope }\end{array}$ & Población \\
\hline Clientes recurrentes & 200 \\
Ejecutivos / Equipos de trabajo & 66 \\
Jefes & 7 \\
Colaboradores & 39 \\
\hline Total & 312 \\
\hline
\end{tabular}

Elaboración propia
Como cirterio de inclusión se consideró a personas en calidad de clientes y trabajadores del área de operaciones y sub áreas dependientes de la misma: 1) clientes, son los que clientes y/o pacientes afiliados a la clínica, y hacen uso reiterado de los servicios, más de dos al mes; 2) jefes, son los tienen más de siete años de experiencia en la clínica, tienen estudios especializados de acuerdo a los requerimientos de la clínica, conocen totalmente los procesos administrativos, resuelven problemas administrativos complicados y brindan soporte a todos los ejecutivos; 3) ejecutivos, son las personas que tienen más de dos años de experiencia en atención al cliente, con estudios universitarios de contabilidad y administración, brindan toda la atención de servicio al cliente, son la imagen de la clínica y están en contacto directo con el cliente y 4) colaboradores son aquellas personas que ayudan a dar el servicio médico como por ejemplo: técnicos enfermeros, y personas quienes facilitan el trabajo a los ejecutivos por ejemplo: logística, facturadores, asistentes, y no están en contacto directo con los cliente. En consecuencia los trabajadores que se excluyen de este estudio es personal que pertenece a otras áreas como: citas, contabilidad, informática, limpieza y área médicas como: jefes de médicos, doctores de consultorios, licenciados del área hospitalaria, etc.

\section{Instrumento}

La técnica de recolección de datos fue la encuesta. El instrumento utilizado es Evaluación de 360 grados del desempeño laboral. Castañeda, De la Torre, Morán y Lara (2002) mencionan que en algunos casos los instrumentos necesarios ya fueron diseñados por otros investigadores y lo único que se requiere es adaptarlos o simplemente adoptarlos. Para fines de esta investigación se adaptó el instru- 
mento para medir el desempeño laboral de los trabajadores de la Clínica Good Hope, el cual consta de 57 ítems. Se validó la confiabilidad del instrumento obteniéndose un coeficiente de 0.90 .

\section{Resultados}

En la presente sesión se evidencian los resultados que demanda la investigación. En primer lugar, se estudió las condiciones sociodemográficas del trabajo. En segundo lugar, se trabajó con las dimensiones de la variable objeto de estudio a fin de demostrar la evaluación de 360 grados del desempeño laboral en el personal que labora en el área de operaciones de la Clínica Good Hope.

\section{Condiciones demográficas}

Tabla 4

Cargo, género y nivel de estudios

\begin{tabular}{|c|c|c|c|}
\hline & & Frecuencia & Porcentajes \\
\hline \multirow{5}{*}{ Cargo } & Jefe & 7 & $2,9 \%$ \\
\hline & Ejecutivo & 66 & $27,0 \%$ \\
\hline & Colaborador & 39 & $16,0 \%$ \\
\hline & Cliente & 132 & $54,1 \%$ \\
\hline & Total & 244 & $100,0 \%$ \\
\hline \multirow{3}{*}{ Género } & Masculino & 133 & $54,5 \%$ \\
\hline & Femenino & 111 & $45,5 \%$ \\
\hline & Total & 244 & $100,0 \%$ \\
\hline \multirow{6}{*}{$\begin{array}{c}\text { Grado } \\
\text { académico }\end{array}$} & Primaria & 0 & $0,0 \%$ \\
\hline & Secundaria & 2 & $0,8 \%$ \\
\hline & Instituto & 46 & $18,9 \%$ \\
\hline & Universidad & 183 & $75,0 \%$ \\
\hline & Posgrado & 13 & $5,3 \%$ \\
\hline & Total & 244 & $100,0 \%$ \\
\hline
\end{tabular}

En la Tabla 4, el $54 \%$ de los encuestados son clientes, los cuales periódicamente se atienden en la clínica. El 27 \% son ejecutivos, quienes brindan el servicio de atención administrativa al clien- te en la Clínica Good Hope. El 16\% son colaboradores quienes brindan atención médica al cliente. El 3 \% son jefes, cuya función es supervisar las tareas que los ejecutivos realizan. El $55 \%$ pertenece al género masculino y el $46 \%$ es femenino, resaltando la gran importancia del liderazgo laboral de las mujeres. El $75 \%$ del personal encuestado tienen grado universitario, el $19 \%$ son egresados de institutos superiores. El $5 \%$ del personal cuentan con nivel de posgrado, que permite a la clínica tener un soporte técnico científico (ver tabla 4).

Tabla 5

Evaluación del desempeño laboral

\begin{tabular}{llll}
\hline & & Frecuencia & Porcentajes \\
\cline { 2 - 4 } Jefe & Bajo & 0 & $0.0 \%$ \\
& Medio & 2 & $28.6 \%$ \\
& Alto & 5 & $71.4 \%$ \\
& Total & 7 & $100.0 \%$ \\
& Bajo & 19 & $28.8 \%$ \\
& Medio & 23 & $34.8 \%$ \\
& Alto & 24 & $36.4 \%$ \\
& Total & 66 & $100.0 \%$ \\
& Bajo & 15 & $38.5 \%$ \\
& Medio & 21 & $53.8 \%$ \\
& Alto & 3 & $7.7 \%$ \\
& Total & 39 & $100.0 \%$ \\
\hline
\end{tabular}

En la tabla 5, se evidencia que el $71 \%$ de los y el $29 \%$, en un nivel medio. En cuanto a los ejecutivos, el $36 \%$ perciben el desempeño laboral en un nivel alto; el $35 \%$ en un nivel medio y el $29 \%$ en un nivel bajo. El 38.5\% de los colaboradores de médico, perciben el desempeño en un nivel bajo, el $53.8 \%$ en un nivel medio $y$, el $8 \%$ en un nivel alto 
Tabla 6

Percepcion de los clientes evaluación de 360 grados

\begin{tabular}{|c|c|c|c|c|c|c|}
\hline & Nunca & Casi nunca & $\begin{array}{l}\text { Algunas } \\
\text { veces }\end{array}$ & $\begin{array}{l}\text { Casi } \\
\text { siempre }\end{array}$ & Siempre & Total \\
\hline $\begin{array}{l}\text { 1. El ejecutivo le comunica y facilita adecua- } \\
\text { damente y a tiempo los procesos, horarios, } \\
\text { nombre de delegados de áreas cuando lo } \\
\text { require. }\end{array}$ & $0.0 \%$ & $18.2 \%$ & $80.3 \%$ & $1.5 \%$ & $0.0 \%$ & $100.0 \%$ \\
\hline $\begin{array}{l}\text { 2. El ejecutivo le proyecta altos principios } \\
\text { éticos. }\end{array}$ & $0.0 \%$ & $0.0 \%$ & $4.5 \%$ & $95.5 \%$ & $0.0 \%$ & $100.0 \%$ \\
\hline $\begin{array}{l}\text { 3. El ejecutivo que lo atiende colabora, es } \\
\text { respetuoso, cordial y actúa anticipadamente } \\
\text { a sus requerimientos. }\end{array}$ & $0.0 \%$ & $0.0 \%$ & $100.0 \%$ & $0.0 \%$ & $0.0 \%$ & $100.0 \%$ \\
\hline 4. Recibe el servicio que requiere. & $0.0 \%$ & $0.0 \%$ & $83.3 \%$ & $16.7 \%$ & $0.0 \%$ & $100.0 \%$ \\
\hline $\begin{array}{l}\text { 5. Ha registrado algún reconocimiento y } \\
\text { felicitaciones hacia algún trabajador. }\end{array}$ & $81.8 \%$ & $2.3 \%$ & $15.9 \%$ & $0.0 \%$ & $0.0 \%$ & $100.0 \%$ \\
\hline
\end{tabular}

En la tabla 6, el $80 \%$ de los clientes percibe que algunas veces el ejecutivo le comunica y facilita adecuadamente a tiempo los procesos, horarios, nombre de delegados de áreas cuando lo requiere, mientras que $18 \%$ casi nunca, y el $2 \%$ casi siempre. Incide no favorablemente en la evaluación de 360 grados y su relación con el desempeño laboral.

El 95\% de los clientes consideran que casi siempre, los ejecutivos muestran y proyectan sus altos principios éticos. Mientras que el 5\% algunas veces. Reflejando de esta manera que la mayoría de clientes toman en cuenta y perciben que los principios éticos juegan un papel importante en la evaluación de 360 grados.

El $100 \%$ de los clientes encuestados consideran que el ejecutivo que lo atiende es respetuoso, cordial y actúa anticipadamente a sus requerimientos.

Así mismo, el 83\% de los clientes manifestaron que algunas veces reciben del personal el servicio que requieren. Lo que significa que los clientes no tienen una clara percepción sobre la modalidad de evaluación de 360 grados y el desempeño laboral del personal.
Por otro lado, el $82 \%$ de los clientes mencionan que nunca recibieron ningún reconocimiento del personal con quien tienen relación de servicio médico o comercial (ver tabla 6).

Tabla 7

Nivel de desempeño del conocimiento del trabajo

\begin{tabular}{cccc}
\hline & & Frecuencia & Porcentajes \\
\hline \multirow{3}{*}{ Jefe } & Bajo & 0 & $0.0 \%$ \\
& Medio & 2 & $28.6 \%$ \\
& Alto & 5 & $71.4 \%$ \\
& Total & 7 & $100.0 \%$ \\
& Bajo & 23 & $34.8 \%$ \\
& Medio & 19 & $28.8 \%$ \\
& Alto & 24 & $36.4 \%$ \\
& Total & 66 & $100.0 \%$ \\
& Bajo & 10 & $25.6 \%$ \\
& Medio & 25 & $64.1 \%$ \\
& Alto & 4 & $10.3 \%$ \\
& Total & 39 & $100.0 \%$ \\
\hline
\end{tabular}

En la tabla 7, el 71\% de los jefes encuestados en la clínica Good Hope perciben el conocimiento de trabajo en un nivel alto, conocen los detalles y procesos del trabajo que realiza, las metas y ob- 
jetivos que persigue la clínica. El $28.6 \%$ percibe en un nivel medio. Con respecto a los ejecutivos, el $36.4 \%$ percibe en un nivel alto; el $28.829 \%$ en un nivel medio y el $34.8 \%$, en un nivel bajo.

En cuanto a los colaboradores, el $64.1 \%$ perciben que el conocmiento del trabajo está en un nivel medio; el $25.5 \%$ en un nivel bajo; mientras que el $10 \%$, en un nivel alto.

Tabla 8

Calidad de trabajo

\begin{tabular}{lccc}
\hline & & Frecuencia & Porcentajes \\
\hline \multirow{3}{*}{ Jefe } & Bajo & 0 & $0.0 \%$ \\
& Medio & 2 & $28.6 \%$ \\
Ejecutivo & Alto & 5 & $71.4 \%$ \\
& Total & 7 & $100.0 \%$ \\
& Bajo & 15 & $22.7 \%$ \\
& Medio & 30 & $45.5 \%$ \\
& Alto & 21 & $31.8 \%$ \\
& Total & 66 & $100.0 \%$ \\
& Bajo & 25 & $64.1 \%$ \\
& Medio & 9 & $23.1 \%$ \\
& Alto & 5 & $12.8 \%$ \\
& Total & 39 & $100.0 \%$ \\
\hline
\end{tabular}

En la tabla 8, el 71\% de los jefes, perciben a la calidad en un nivel alto, es decir, aquí el personal frecuentemente se autoevalúa y motiva constantemente a sus pares para satisfacer al cliente; el $28 \%$ en un nivel medio, es decir desconocen el $100 \%$ de las características del servicio al cliente y calidad de trabajo.

Con respecto a los ejecutivos, el $45.5 \%$ percibe la calidad de trabajo en un nivel es medio; el $31.8 \%$, en un nivel alto y el $22.7 \%$, en un nivel bajo.

Según los colaboradores, el $64.1 \%$ percibe la calidad de trabajo en un nivel es bajo; el $12.8 \%$ en un nivel alto; mientras que el $23.1 \%$, en un nivel medio.

Tabla 9

Relaciones con las personas

\begin{tabular}{lccc}
\hline & & Frecuencia & Porcentajes \\
\hline \multirow{3}{*}{ Jefe } & Bajo & 2 & $28.4 \%$ \\
& Medio & 0 & $0.0 \%$ \\
& Alto & 5 & $71.6 \%$ \\
& Total & 7 & $100.0 \%$ \\
& Bajo & 21 & $31.8 \%$ \\
& Medio & 20 & $30.3 \%$ \\
& Alto & 25 & $37.9 \%$ \\
& Total & 66 & $100.0 \%$ \\
& Bajo & 35 & $89.7 \%$ \\
& Medio & 1 & $2.6 \%$ \\
& Alto & 3 & $7.7 \%$ \\
\hline
\end{tabular}

En la tabla 9, el $71.6 \%$ de los jefes, perciben las relaciones con la personas en un nivel alto, debido a una comunicación asertiva; el $28.4 \%$ en un nivel bajo. Respecto a los ejecutivos, el $30.3 \%$ en un nivel medio; el $37.9 \%$, en un nivel alto y el $31.8 \%$, en un nivel bajo. Según, los colaboradores, el 89.7 \% percibe las relaciones humanas en un nivel bajo; el $7.7 \%$ en un nivel alto; mientras que el $2.6 \%$, en un nivel medio. 
Tabla 10

Estabilidad emotiva

\begin{tabular}{lccc}
\hline & & Frecuencia & Porcentajes \\
\hline \multirow{3}{*}{ Jefe } & Bajo & 2 & $29.6 \%$ \\
& Medio & 0 & $10.0 \%$ \\
& Alto & 5 & $60.4 \%$ \\
& Total & 7 & $100.0 \%$ \\
& Bajo & 20 & $29.3 \%$ \\
& Medio & 21 & $31.8 \%$ \\
& Alto & 25 & $36.9 \%$ \\
& Total & 66 & $100.0 \%$ \\
& Bajo & 36 & $92.3 \%$ \\
& Medio & 0 & $0.0 \%$ \\
& Alto & 3 & $7.7 \%$ \\
& Total & 39 & $100.0 \%$ \\
\hline
\end{tabular}

En la tabla 10, el $60.4 \%$ de los jefes, perciben estabilidad emotiva en un nivel alto; el $29.6 \%$ en un nivel bajo y el $10.0 \%$, en un nivel medio. Respecto a los ejecutivos, el $31.8 \%$ perciben la estabilidad emotiva en un nivel medio; el $36.9 \%$, en un nivel alto y el $29.3 \%$, en un nivel bajo. Según, los colaboradores, el $92.3 \%$ percibe la estabilidad emotiva en un nivel bajo; el $7.7 \%$ en un nivel alto.

Tabla 11

Capacidad de síntesis

\begin{tabular}{llcc}
\hline & & Frecuencia & Porcentajes \\
\hline \multirow{4}{*}{ Jefe } & Bajo & 2 & $21.6 \%$ \\
& Medio & 0 & $7.0 \%$ \\
& Alto & 5 & $71.4 \%$ \\
& Total & 7 & $100.0 \%$ \\
& Bajo & 29 & $43.9 \%$ \\
& Medio & 15 & $22.7 \%$ \\
& Alto & 22 & $33.3 \%$ \\
& Total & 66 & $100.0 \%$ \\
& Bajo & 36 & $92.3 \%$ \\
& Medio & 0 & $0.0 \%$ \\
& Alto & 3 & $7.7 \%$ \\
& Total & 39 & $100.0 \%$ \\
\hline
\end{tabular}

En la tabla 11, el $71.4 \%$ de los jefes, perciben la capacidad de síntesis en un nivel alto; el $29.6 \%$ en un nivel bajo y el $7.0 \%$, en un nivel medio y el 21.6 en un nivel bajo. Respecto a los ejecutivos, el $33.3 \%$ perciben la capacidad de síntesis en un nivel alto; el $22.7 \%$, en un nivel medio y el $43.9 \%$, en un nivel bajo. Según, los colaboradores, el 7.7 \% percibe la capacidad de síntesis en un nivel bajo y el $92.3 \%$ en un nivel bajo.

Tabla 12

Capacidad de análisis

\begin{tabular}{llcc}
\hline & & Frecuencia & Porcentajes \\
\hline \multirow{4}{*}{ Jefe } & Bajo & 2 & $26.4 \%$ \\
& Medio & 0 & $5.4 \%$ \\
& Alto & 5 & $69.2 \%$ \\
& Total & 7 & $100.0 \%$ \\
& Bjecutivo & 26 & $39.4 \%$ \\
& Medio & 17 & $25.8 \%$ \\
& Alto & 23 & $34.8 \%$ \\
& Total & 66 & $100.0 \%$ \\
& Bajo & 36 & $92.3 \%$ \\
& Medio & 1 & $2.6 \%$ \\
& Alto & 2 & $5.1 \%$ \\
& Total & 39 & $100.0 \%$ \\
\hline
\end{tabular}

En la Tabla 12, el $69.2 \%$ de los jefes, perciben la capacidad de análisis en un nivel alto; el $5.4 \%$, en un nivel medio y el $26.4 \%$, en un nivel bajo. Respecto a los ejecutivos, el $34.8 \%$ perciben la capacidad de síntesis en un nivel alto; el 25.8\%, en un nivel medio y el $39.4 \%$, en un nivel bajo. Según, los colaboradores, el $5.1 \%$ percibe la capacidad de síntesis en un nivel alto; el $2.6 \%$, en un nivel medio y el $92.3 \%$, en un nivel bajo.

\section{Discusión}

En la tesis de Cornelio (2011) titulada: "Evaluación de $360^{\circ}$ del desempeño la- 
boral de los supervisores del área de corte de Industrias Citrícolas de Montemorelos, S.A", se encontró que la aplicación del modelo de evaluación de 360 grados del desempeño laboral en supervisores, jefes y obreros tienen diferente percepción. Estos supervisores se autoevaluaron, y a su vez, fueron evaluados por obreros, supervisores y jefes. El promedio de autoevaluación de los supervisores fue igual a 4.71 y la evaluación promedio de los obreros, jefes y colegas fue igual a 4.23. El resultado evidenció que existe diferencia significativa en la autoevaluación del nivel del desempeño laboral y las demás evaluaciones hechas por los obreros, jefes inmediatos y colegas supervisores; en ese sentido, se encontró correlación significativa. En la presente investigación se encontró que el $71 \%$ de los jefes, el $36 \%$ de los ejecutivos y el $54 \%$ de los colaboradores perciben el desempeño laboral de los ejecutivos en un nivel alto. Este resultado explica que la evaluación de 360 grados permite que el personal de la clínica tenga mayor conocimiento sobre su trabajo, respecto a la calidad de trabajo, relaciones con las personas, estabilidad emotiva del personal, mayor capacidad de síntesis y capacidad de análisis.

En la tesis de Cornejo, Guirola y Pérez (2012) titulada "Modelo de evaluación de 360 grados, para fortalecer el desempeño laboral del personal de la Corporación Salvadoreña de Turismo (CORSATUR), se encontró que la implementación del modelo de evaluación de 360 grados es viable toda vez que permite a la gerencia manejar información en la toma de decisiones para mejorar la eficiencia y eficacia en el desempeño laboral de la empresa. Wolford (2007), en su tesis de investigación titulada: "Diseño de un sistema de evaluación del desempeño del recurso humano y medición de la satisfacción del cliente en la empresa editores siglo veintiuno", se realizó la encuesta a 8 trabajadores evaluadores y a 24 trabajadores evaluados y se logró demostrar que el sistema usado no está acorde a los requerimientos de una buena evaluación del desempeño, el modelo de evaluación no se ajusta a los contenidos de cada puesto de trabajo. Se encontró, en esta, la investigación en la que se aplicó la Evaluación de 360 grados del desempeño laboral en los trabajadores del área de operaciones de la Clínica Good Hope, 2015, niveles de percepción sobre conocimiento sobre el trabajo, calidad del trabajo, relaciones con las personas, estabilidad emotiva, capacidad de síntesis y capacidad de análisis, mediante la perspectiva del jefe, ejecutivos y colaboradores. Sin embargo, la percepción baja sobre capacidad de síntesis, indica que no se acomoda al puesto de trabajo.

\section{Conclusiones}

Mediante la evaluación de 360 grados del desempeño laboral se percibe que los trabajadores del área de operaciones de la Clínica Good Hope, a nivel de jefes y ejecutivos tienen alto desempeño laboral. En cambio se percibe que los colaboradores tienen un desempeño laboral medio.

- La evaluación de 360 grados del desempeño laboral ha permitido conocer que existe conocimiento del trabajo en un nivel alto mediante la percepción de los jefes y ejecutivos. Sin embargo, el personal colaborador percibe el conocimiento del trabajo en un nivel medio.

- La evaluación de 360 grados del desempeño laboral evidencia que los jefes y ejecutivos perciben, en un nivel alto, la calidad del trabajo. Por el contrario, los colaboradores perciben la calidad del trabajo en un ni- 
vel medio; sin embargo, el personal colaborador percibe el conocimiento del trabajo en un nivel medio.

- La evaluación de 360 grados del desempeño laboral, respecto a las relaciones con las personas, evidenció que los jefes y los ejecutivos perciben en un nivel alto, mientras que los colaboradores perciben en un nivel bajo por la existencia de comunicaión poco asertiva.

- La evaluación de 360 grados del desempeño laboral evidencia que los jefes y ejecutivos perciben la estabilidad emotiva en un nivel alto.
Por el contrario, los colaboradores perciben la estabilidad emotiva en un nivel medio, sin embargo, el personal colaborador percibe el conocimiento del trabajo en un nivel bajo.

- La evaluación de 360 grados del desempeño laboral respecto a la capacidad de síntesis evidenció que el los jefes y los ejecutivos perciben en un nivel alto, mientras que los colaboradores perciben en un nivel bajo por las pocas oportunidades de capacitación y especialización. 


\section{Referencias}

Arrigada, R. (2002). Diseño de un sistema de medición de desempeño para evaluar la gestión municipal: una propuesta metodológica.

Recuperado de http://www.eclac.org/publicaciones/xml/3/10883/manual20. pdf

Bernández, M. (2006). Tecnología del desempeño humano. Boston: Global Business Pres.

Chiavenato, I. (2000). Administración de recursos humanos $\left(5^{\mathrm{a}}\right.$ ed.). Bogotá: McGraw Hill

Chiavenato, I. (2001). Gestión del talento humano: el nuevo papel de los recursos humanos en las organizaciones. Bogotá: McGraw-Hill.

Castañeda, J., De la Torre, M., Morán, J., y Lara, L. (2002). Metodología de la investigación. México: McGraw-Hill.

Cornejo, A., Guirola, G. y Pérez, E. (2012). Modelo de evaluación 360 grados, para fortalecer el desempeño laboral del personal de la Corporación Salvadoreña de Turismo (CORSATUR). Tesis de licenciatura. San Salvador: El Salvador.

Cornelio, T. (2011). Evaluación de $360^{\circ}$ del desempeño laboral de los supervisores del área de corte de Industrias Citrícolas de Montemorelos, S.A. Tesis de maestría, Universidad de Montemorelos. México.

Crespo, A., Mortis, A., y Tapia, C. (2011). La realimentación para la mejora del desempeño docente de los profesores auxiliares en una institución de educación superior.

Recuperado de http://www.itson.mx/ publicaciones/pacioli/Documents/ no74/25.__lareal_1.pdf.
Encina, G. (2003). Cultivando la productividad del personal. Facilitando el diálogo entre partes. California.

Faria, F. (1995). Desarrollo organizacional. Enfoque integral. México: Editorial Mc Graw Hill.

García, M. (2001). La importancia de la evaluación de desempeño. Recuperado de http: //www. cem.itesm. $\mathrm{mx} /$ dacs/publicaciones / proy/n9/ exaula/ mgarcia.html.

González, M., y Olivares, S. (2005). Administración de recursos humanos. México: CECSA.

Hellriegel, D., Jackson, S., y Slocum, J. W. (2005). Administración, un enfoque basado en competencias (10 $\mathrm{ed}$.). Buenos Aires: Thomson.

Lara, C., Moras, C., Morales, L., y Galán, J. (2010). Aplicación de la evaluación $360^{\circ}$ para conocer el desempeño de los trabajadores de una empresa metal-mecánica. Revista de la Ingeniería Industrial, 4(1), 1-12.

Lau, J. (2010). Evaluación del desempeño de personal bibliotecario. Buenos Aires: Alfagrama.

Mondy, R. W. y Noé, R. M. (2005). Administración de recursos humanos $\left(9^{\mathrm{a}}\right.$ ed.). México: Prentice Hall.

Nieto, L. V., Mejía, J. A., Rojas, G., Artunduaga, L. A., Villegas, P., Escobar, J., Fernández, H. (2003). Evaluar para mejorar. Recuperado de

Kerlinger, F., \& Lee, H. (2002). Investigación del comportamiento. Métodos de investigación en las ciencias sociales (4ta edición). Mexico: Mcgraw-Hill, Interamericana.

Robbins, S., y Coulter, M. (2005). Administración ( $8^{\mathrm{a}}$ ed.). México: Prentice Hall. 
Robbins S., y Decenzo, A. (2002). Fundamentos de administración ( $3^{\mathrm{a}}$ ed.). México: Prentice Hall.

Werther, W., y Davis, K. (1995). Administración de recursos humanos. El capital humano de las empresas (Sexta edición). México: Editorial: McGraw-Hill Interamericana.
Wolford, M. (2007). Diseño de un sistema de evaluación del desempeño del recurso humano y medición de la satisfacción del cliente en la empresa editores siglo veintiuno. Tesis de Ingeniería. Universidad de San Carlos, Guatemala. 
